\title{
Estimation of effective vaccination rate: pertussis in New Zealand as a case study
}

\author{
A. Korobeinikov ${ }^{\mathrm{a}, *, 1}$, P.K. Maini ${ }^{\mathrm{a}, 2}$, W.J. Walker ${ }^{\mathrm{b}}$ \\ ${ }^{a}$ Centre for Mathematical Biology, Mathematical Institute, University of Oxford, 24-29 St Giles', Oxford OX1 3LB, UK \\ ${ }^{\mathrm{b}}$ Department of Mathematics, University of Auckland, Private Bag 92019, Auckland, New Zealand
}

Received 14 August 2002; received in revised form 5 April 2003; accepted 8 April 2003

\begin{abstract}
In some cases vaccination is unreliable. For example vaccination against pertussis has comparatively high level of primary and secondary failures. To evaluate efficiency of vaccination we introduce the idea of effective vaccination rate and suggest an approach to estimate it. We consider pertussis in New Zealand as a case study. The results indicate that the level of immunity failure for pertussis is considerably higher than was anticipated.

(C) 2003 Elsevier Ltd. All rights reserved.
\end{abstract}

MSC: 92D30; 34D20

Keywords: Mathematical modelling; Endemicity; Childhood infectious diseases; Immunity

\section{Introduction and background}

Vaccination does not necessarily imply life-long immunity for a vaccinated person. In some cases, vaccination is ineffective and fails to provide immunity at all (the so-called "primary failure"); sometimes immunity acquired through vaccination wanes and does not provide life-long protection ("secondary failure"). For example, the New Zealand statistical data show that in the case of measles, when vaccination is known to be reliable, about one-third of those infected, whose vaccination history is known, received one dose, and about $2 \%$ received two doses of the vaccine (NZ ESR, 1997). This is especially relevant to pertussis. The vaccine against pertussis is known to be unreliable: the immunity acquired due to vaccination wanes over time (Cullen and Walker, 1997).

This leads to the question of how effective is the vaccination in reality. To quantitatively describe

\footnotetext{
*Corresponding author.

E-mail addresses: korobein@maths.ox.ac.uk (A. Korobeinikov), maini@maths.ox.ac.uk (P.K. Maini).

${ }^{1}$ Supported by the Foundation for Research, Science and Technology of New Zealand, through Project contract UOXX0101.

${ }^{2}$ Partly supported by a Royal Society Leverhulme Trust Senior Fellowship.
}

efficiency of vaccination we introduce the term effective vaccination rate. By this term we mean the portion of the population which at a given moment has immunity acquired through the vaccination. If $N$ is the total population, $n(a)$ the population of age $a$ and $P(a)$ the portion of population of age $a$ immune through the vaccination, then the effective vaccination rate, $\hat{p}$, is

$\hat{p}=\frac{\int_{0}^{\infty} P(a) n(a) \mathrm{d} a}{N}$.

The effective vaccination rate reflects the whole population and takes into account both primary and secondary vaccination failure without distinguishing between them. Humans are assumed to be born susceptible; at some age a fraction of them is vaccinated (but because of the primary vaccination failure even the maximum vaccination level is lower than the rate of vaccination). After some time immunity starts to wane, and the fraction of people immune due to vaccination declines. Fig. 1 schematically represents vaccination and the immunity fadeout by age.

For obvious reasons, the effective vaccination rate cannot be measured directly since it implies continual testing of a significant portion of the population. The cost of such mass tests can be considerably higher than that of the vaccination campaign itself, and it definitely 


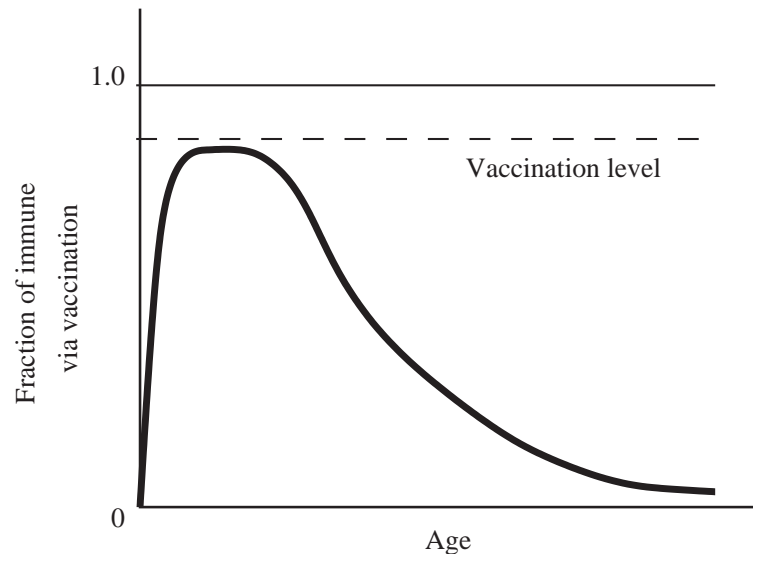

Fig. 1. Schematic distribution of population preserving immunity acquired through vaccination.

would cause great public concern. For this reason, indirect methods for evaluation of the effectiveness of vaccination are needed. In this work, we attempt to evaluate the effective vaccination rate for pertussis in New Zealand using a simple and reliable approach based on published statistical data.

Pertussis, or whooping cough, is a highly contagious disease of the respiratory tract, spread by droplets. Infection is particularly severe in young infants, especially for those under 1-year old, a group which is most likely to be hospitalized. Pertussis mortality rates are higher in the first year of life than at any other age; beyond 3 years of age mortality rates are relatively low.

In comparison with other common infectious illnesses, such as mumps and measles, descriptions of pertussis appeared relatively recently, in the 16th century. The disease was known under different names: Italians spoke of the "dog bark", in England it was known as the "chin cough" or "kin cough", later to be called whooping cough, the Chinese called it the " 100 day cough" because of the protracted course of the disease. Thomas Sydenham first used the term infantum pertussis in 1670, "pertussis" meaning a violent cough of any kind. Bordet and Gengou identified the causative organism in 1906 (hence the name Bordetella pertussis), and the first crude vaccine was developed soon after from killed bacteria.

In New Zealand, monovalent pertussis vaccine was introduced by the Department of Health in 1945, and from 1953 it was also available in combination with diphtheria vaccine. Routine childhood immunization started in 1960. Three doses were given at 3, 4 and 5 months of age, respectively. In 1971, the policy was altered to two doses of vaccine given at 3 and 5 months of age. Following this schedule change there was a progressive increase in hospitalizations in 1974, 1978 and 1982, and consequently a third dose given at 6 weeks of age was added in 1984 to provide earlier protection. A fourth dose of pertussis vaccine was added in 1996 given at 15 months of age with the goals of increasing protection in young children and reducing risk of transmission to younger siblings. A further booster dose given at 4 years of age was introduced in February 2002 to protect children during the early school years and to decrease transmission of the infection to younger children.

Nevertheless, the current situation with pertussis in New Zealand can hardly be considered as satisfactory. Recurrent epidemics occur every 4-5 years, with high numbers of infants hospitalized. The most recent epidemics of pertussis occurred in 1999-2001. Since the start of the epidemic in June 1999 and by the end of March 2001, a total of 5781 cases (of which 2737 cases were laboratory confirmed) have been notified (NZ Ministry of Health, 2001). There have been 412 hospitalizations, and one death was reported (NZ Ministry of Health, 2001). Also there have been 550 hospitalizations during the epidemics in 1996 (NZ Health Information Service, 2000).

\section{Model}

There is no specific limitation on the type of model for this work; in fact any reasonably accurate model can be used. We will use a discrete-time compartmental model. The main advantage of the discrete-time models compared with continuous-time models is their natural time-scaling. The natural time-scale for epidemiological processes is a parasite generation, and we shall use this as the time interval. By the term "parasite generation" we mean the average time interval which commences when a susceptible host is exposed to an infective dose, includes the period during which the host passes infection and ends when the host is fully recovered, isolated or dead.

The choice of a parasite generation as the time interval for a discrete-time model leads to important consequences. Firstly, with a parasite generation as the time interval we naturally approximate the recovery process by a step function (Fig. 2, curve A, B), whereas for continuous-time models, unless integro-differential equations or equations with a time delay are used, one has to postulate that motion from exposed to the infectious class and then to the recovery class occurs at constant rates (Fig. 2, curve C). This last assumption, while mathematically convenient, is rarely realistic and can lead to results contradicting observations (Keeling and Grenfell, 1997). Secondly, since in the case of a discrete-time model we consider disease transmission not as a continuous process but in terms of secondary cases produced by an infective for a parasite generation, we have no need for an exposed class, or for any class for the hosts in the incubation or in the latent state. The third (but not the least important) advantage provided 


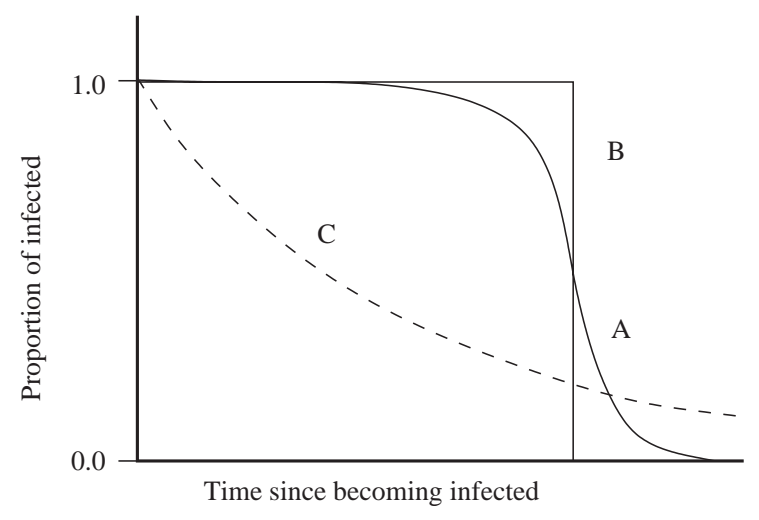

Fig. 2. Schematic representation of recovery. Here the curve $A$ is for observed data, the curve B is for recovery of all infected after a definite period of time (a parasite generation) and the curve $\mathrm{C}$ is for continuous-rate recovery. A model has a basic reproductive number $R_{0}$ proportional to the area under the curve.

by a discrete-time model with a parasite generation as the time interval, is that it allows natural interpretation of all model parameters and data obtained. The last feature is of definite importance for this work, and determined our choice of modelling approach.

An apparent drawback of the discrete-time models is that parasite generations can overlap. Nevertheless, this does not affect the inter-epidemic period.

We consider a SIR compartment model, that is we assume that a population of size $N$ is partitioned into susceptibles $S$, infected $I$, and immune (or removed) $R$ compartments, i.e. $N=S+I+R$. We will denote the number of individuals in a compartment in a generation by a capital letter with a subindex, e.g. $I_{n}, S_{n+1}$, etc. All newborns come into the susceptible compartment. After infection, an individual moves from the susceptible class into the infected class and then into the immune compartment as a result of recovery, death or isolation. We assume here that recovery implies life-long immunity, and hence there is no return from the immune compartment into the susceptible compartment.

The population size $N$ is assumed constant, and we neglect the susceptibles' natural mortality. The latter assumption is safe, since for pertussis, as well as for the majority of childhood diseases, the class of susceptibles is composed of young people, and in developed countries child mortality is low. Nevertheless, the susceptibles' mortality must be taken into account if a developing country, where child mortality is high, is under consideration.

By the principle of mass action, we assume that during a parasite generation an average infective comes into infective contact with and might eventually infect $R_{0}$ people (susceptible, infected, or recovered), where $R_{0}$ is the basic reproduction number. Some of these people may be already infected or recovered and therefore clinically unaffected by the contact. Then, assuming that the number of infectives in the $(n+1)$-th generation is equal to the number of infective contacts, we obtain the equations

$S_{n+1}=S_{n}-R_{0} I_{n} \frac{S_{n}}{N}+b N, \quad I_{n+1}=R_{0} I_{n} \frac{S_{n}}{N}$.

The constant population size assumption allows us to omit the third equation,

$R_{n+1}=R_{n}+I_{n}-b N$,

for the immune population.

The principle of mass action is deficient in that for a finite time interval a susceptible may be infected by more than one infective and will be counted eventually as several infectives in the next generation (Cullen et al., 1999, 2003). Furthermore, system (1) is structurally unstable: the phase trajectories of the system near the fixed point form closed loops (Fig. 3), a structure which is fragile. In spite of the above-mentioned deficiencies, the model allows us to determine the inter-epidemic period of an endemic disease, and thereby to estimate the effective vaccination rate.

System (1) has a unique fixed point, an endemic equilibrium state with coordinates

$S^{*}=N / R_{0}, \quad I^{*}=b N$.

A substitution $x=S / S^{*}$ and $y=I / I^{*}$ leads to the system

$x_{n+1}=\rho+x_{n}-\rho x_{n} y_{n}, \quad y_{n+1}=x_{n} y_{n}$.

The system depends on the parameter $\rho=R_{0} b$ only.

\section{Inter-epidemic period}

We now apply standard linear analysis techniques (see e.g. Murray, 1993; Tu, 1994). The linear approximation of system (2) near the fixed point is

$\eta_{n+1}=(1-\rho) \eta_{n}-\rho \xi_{n}, \quad \xi_{n+1}=\eta_{n}+\xi_{n}$,

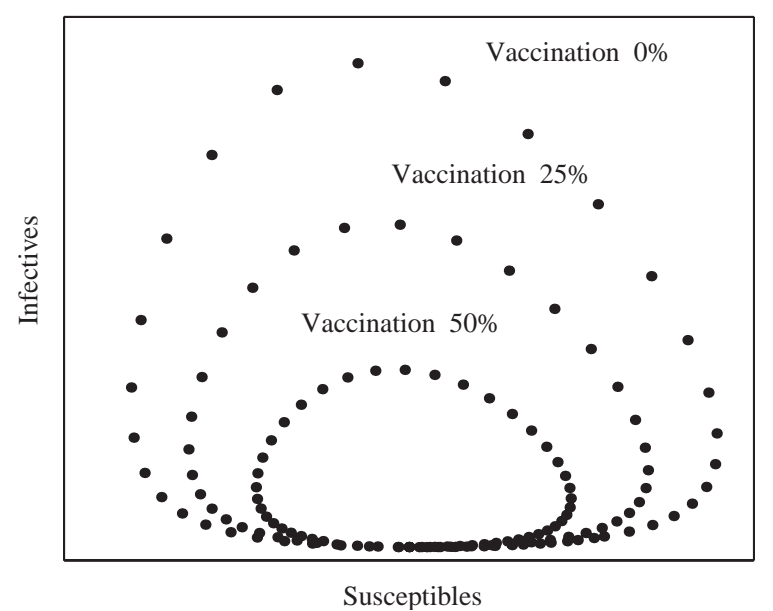

Fig. 3. Typical phase orbits of system (1) for different vaccination rates and the same initial conditions (here $b=\frac{1}{480}$ and $R_{0}=16$ ). 
where $\eta=x-1$ and $\xi=y-1$. The characteristic equation of the linearized system is

$\lambda^{2}-(2-\rho) \lambda+1=0$

where

$\lambda_{1,2}=\frac{2-\rho}{2} \pm \frac{1}{2} \sqrt{\rho^{2}-4 \rho}$.

System (2) depends on the parameter $\rho=R_{0} b$ only. If $\rho=R_{0} b<4$ (a condition which holds for all childhood infections) then the eigenvalues $\lambda_{1}$ and $\lambda_{2}$ are complex conjugates and their absolute values are equal to one exactly, $\left|\lambda_{1,2}\right|=1$. In this case, the linearized system (3) defines an elliptic rotation, a linear transformation of the $\eta \xi$ plane similar to a dilation by a factor of $|\lambda|=1$ with a rotation through the angle

$\theta=\arg \lambda=\arctan \frac{\sqrt{4 \rho-\rho^{2}}}{2-\rho}$.

If $\rho=R_{0} b>4$, a condition which is hardly possible in reality, then both eigenvalues of the linearized system (3) are real and negative, and $\lambda_{1}<-1<\lambda_{2}<0$. The fixed point is a saddle point in this case.

Since the system, and hence the angle $\theta$, depends on the parameter $\rho=R_{0} b$ only, the inter-epidemic period does not depend on the population size $N$. This conclusion is supported by statistical data which demonstrate that prior to mass vaccination in countries with different population sizes but with similar birth rates, e.g. in New Zealand, England and United States (NZ Yearbook, 1998; US Bureau of Census, 2002), the inter-epidemic periods of such diseases as measles (Anderson and May, 1991; Bartlett, 1957, 1960; Cullen and Walker, 1996; Soper, 1929) and pertussis (Anderson and May, 1991; Cullen and Walker, 1997; Anderson et al., 1984) were the same.

Due to low birth rate, for humans the parameter $\rho=$ $R_{0} b$ is fairly small: for the majority of human diseases, including pertussis and measles, $\rho \sim 10^{-2}$. If $\rho \ll 1$, then $\frac{\sqrt{4 \rho-\rho^{2}}}{2-\rho} \approx \frac{2 \sqrt{\rho}}{2}=\sqrt{\rho}$ and $\theta \approx \arctan \sqrt{\rho} \approx \sqrt{\rho}$,

and hence the inter-epidemic period

$T_{n} \approx \frac{2 \pi}{\sqrt{\rho}}$.

(For a discrete-time system a period is not a time interval, as for continuous-time models, but a number of discrete intervals - parasite generations. We are using the notation $T_{n}$ for the discrete-time systems to stress the difference.)

For pertussis $R_{0} \approx 16$ (Anderson and May, 1991, p. 70), and there are about 12 parasite generations per year (Anderson and May, 1991, p. 31). Prior to mass vaccination the birth rate in New Zealand, United States and United Kingdom was about 25 births per thousand of the population per year (NZ Yearbook, 1998; US
Bureau of Census, 2002). These figures give $b=\frac{1}{480}$ and $\rho=\frac{1}{30}$, and hence Eq. (4) gives the period $T_{n}=34.4$ parasite generations, or about a 3-year cycle. Prevaccination statistics from New Zealand (Cullen and Walker, 1997) and from England and Wales (Anderson and May, 1991; Anderson et al., 1984) also give the 3-year (156 weeks) cycle for pertussis.

Computations demonstrate that the inter-epidemic period increases with amplitude. Table 1 contains data from computations for $\rho=\frac{1}{30}$ (the figure for pertussis in New Zealand, United Kingdom and United States prior to mass vaccination) for different amplitudes. These data indicate that, at least for amplitudes such that $I_{\max } / I_{\min } \leqslant 4$, Eq. (4) provides a good approximation of the period $T_{n}$ and that the period extends as the level of infection grows.

Statistical data demonstrate that in the pre-vaccination period low-amplitude oscillations were typical for pertussis. For example, in England and Wales the ratio $I_{\max } / I_{\min }$ was about $4-4.5$ (Anderson and May, 1991, p. 135). For such amplitudes, the computed period practically coincides with the value given by Eq. (4).

To verify that this fit of the predicted inter-epidemic period with the clinical data is not a coincidence we can consider other childhood infections. Measles has $R_{0} \approx 14$ and a parasite generation of 14 days on average (Anderson and May, 1991), i.e. there are 26 parasite generations per year. Hence, for England, United States and New Zealand in the pre-vaccination period $\rho \approx 0.014$, and $T_{n} \approx 53$ parasite generations. This figure fits well with clinical observations: after the Second World War and prior to the introduction of mass vaccination, measles epidemics occurred in England and

Table 1

Different amplitudes obtained by varying initial conditions. Period $T_{n}$ vs. amplitudes for $\rho=\frac{1}{30}$ (Eq. (4) gives $T_{n}=34.4$ )

\begin{tabular}{|c|c|c|c|c|c|}
\hline$\frac{I_{\min }}{I^{*}}$ & $\frac{I_{\max }}{I_{\min }}$ & $T_{n}$ & $\frac{I_{\min }}{I^{*}}$ & $\frac{I_{\max }}{I_{\min }}$ & $T_{n}$ \\
\hline 0.002 & 4180 & 49 & 0.22 & 12.5 & $36-37$ \\
\hline 0.004 & 1879 & 47 & 0.24 & 11 & 36 \\
\hline 0.006 & 1175 & 46 & 0.26 & 9.8 & 36 \\
\hline 0.008 & 848 & 45 & 0.28 & 8.75 & 36 \\
\hline 0.01 & 645 & 45 & 0.30 & 7.9 & 36 \\
\hline 0.02 & 285 & 43 & 0.35 & 6.2 & $35-36$ \\
\hline 0.03 & 173 & 42 & 0.40 & 5.0 & $35-36$ \\
\hline 0.04 & 121 & 41 & 0.45 & 4.2 & 35 \\
\hline 0.05 & 91 & 40 & 0.50 & 3.5 & 35 \\
\hline 0.06 & 73 & 40 & 0.55 & 3 & 35 \\
\hline 0.07 & 59 & $39-40$ & 0.60 & 2.58 & 35 \\
\hline 0.08 & 50 & 39 & 0.65 & 2.25 & 35 \\
\hline 0.09 & 43 & 39 & 0.70 & 1.97 & $34-35$ \\
\hline 0.10 & 37 & $38-39$ & 0.75 & 1.73 & $34-35$ \\
\hline 0.12 & 29 & 38 & 0.80 & 1.54 & 34-35 \\
\hline 0.14 & 24 & $37-38$ & 0.85 & 1.38 & $34-35$ \\
\hline 0.16 & 19.9 & 37 & 0.90 & 1.24 & 34-35 \\
\hline 0.18 & 16.6 & 37 & 0.95 & 1.1 & 34-35 \\
\hline 0.20 & 14.3 & 37 & 0.999 & 1.002 & $34-35$ \\
\hline
\end{tabular}


Wales with a 2-year period (Anderson and May, 1991, p. 131); 2-year epidemic cycles were also typical for New Zealand (Cullen and Walker, 1996) and USA (US Department of Human Services, 1994). African states, such as Cameroun, 1968-1975, and Nigeria, 1958-1961, provide examples of 1-year measles epidemic cycles (Anderson and May, 1991, p. 129), which is in agreement with the birth rate of more than 50 births per thousand of the population recorded in these countries (US Bureau of Census, 2002).

However, measles perhaps is not the best example, as it exhibits a strong seasonality in incidence number (Anderson and May, 1991; Cullen et al., 2003; Dietz, 1976; Keeling and Grenfell, 1997). This seasonality may break the natural epidemic cycle adjusting it to the seasons. Mumps has $R_{0}=12-13$ and an average parasite generation of 21 days (Anderson and May, 1991), or 17-18 parasite generations per year. For England and Wales, these figures give $\rho=0.018$ and $T_{n}=47$ parasite generations, i.e. 3-year cycles which coincides with the observations (Anderson et al., 1984). Three-year cycles were also observed in the prevaccination era in the USA (US Department of Human Services, 1994). That is we have another fit between the model prediction and the observations.

Prior to the introduction of mass vaccination against poliomyelitis, a 3-year cycle was typical in the United States (US Department of Human Services, 1994). For poliomyelitis in the United States $R_{0}=6$ (Anderson and May, 1991, p. 70) and there are about 20 parasite generations in a year. These figures give $\rho \approx 0.0075$ and $T_{n}=72.6$ which is higher than the expected 60 parasite generations, but it still fits data for England and Wales (3-5 years cycles).

These examples demonstrate a good correlation between the inter-epidemic periods predicted and the available statistical data. This remarkably good fit is unlikely to be accidental, and it shows that the inter-epidemic period is an important and reliable indicator.

\section{Vaccination impact}

Effect of vaccination of a constant fraction, $p$, of the newborns is equivalent to direct decline of influx of new susceptibles into the system. Vaccinated individuals go directly into the immune compartment and, if their immunity is preserved, do not participate in further events. If a constant fraction $p$ of the newborns is vaccinated then the system behaviour is equivalent to that of system (1) with the apparent susceptibles influx rate $\hat{b}=(1-p) b$.

Consequently, the mass vaccination results in decline of the endemic equilibrium level of the infectives $I^{*}$ and, according to Eq. (4), in extension of the inter-epidemic period $T_{n}$. A lower value of the infectives' equilibrium level $I^{*}$ also suggests that the vaccination will result in a lower level of the infective maxima (see Fig. 3).

The available statistical data support both these conclusions. The most remarkable success of mass vaccination is the complete world-wide elimination of smallpox. Although complete elimination has not been achieved for other endemic infections, decline of the infection levels accomplished by prolonging of the interepidemic intervals has been observed for all diseases after mass vaccination was introduced.

For example, in the United States after the introduction of mass vaccination the 2-year cycle of measles turned into 4-6-year cycles and the infection level dropped by about ten times (US Department of Human Services, 1994). According to Eq. (4), three times longer inter-epidemic period (6 years instead of 2 years) implies nine times lower susceptibles influx, i.e. the rate of successful vaccination is about $85-90 \%$ of the newborn. A similar result - a 9-year inter-epidemic period and a decline of the infection level of about ten times - has been achieved in the US for rubella (US Department of Human Services, 1994). Mass vaccination against mumps also was accompanied by a decline of the infectives' level of about ten times and prolongation of the inter-epidemic period to 7 years (US Department of Human Services, 1994) (perhaps even longer, since the available time series are not long enough). Similar results have been observed in other countries and for other diseases.

We can estimate the effective vaccination rate comparing the real observed inter-epidemic period with that provided by Eq. (4). According to Eq. (4), the apparent rate of new susceptibles influx into the susceptibles compartment, $\hat{b}$, connected with the interepidemic period $T_{n}$, is given by

$\hat{b}=\frac{4 \pi^{2}}{R_{0} T_{n}^{2}}$.

Comparing $\hat{b}$ with the actual birth rate, $b$, we obtain the effective vaccination rate

$\hat{p}=\frac{b-\hat{b}}{b}=1-\frac{\hat{b}}{b}$.

Available data for pertussis from New Zealand, England, Wales and the United States exhibit lengthening of the inter-epidemic periods and (at least temporary) decline of the infective maxima levels after mass vaccination was introduced. In New Zealand, the first mass vaccination schedule was put into place in 1960 . During 1962-1976 there were observed 3-4 year cycles with amplitudes lower than prior to mass vaccination (Cullen and Walker, 1997). The average period of 180 weeks, or $T_{n} \approx 41.5$ parasite generations, corresponds to $\hat{b} \approx 0.00143$, whereas the actual birth rate in 
New Zealand in this period was 22 births per thousand mean population annually (NZ Yearbook, 1998, p. 92), or $b \approx 0.00183$. Comparing the actual birth rates with the apparent birth rate $\hat{b}$, we obtain that effective vaccination rates in New Zealand in this period were about 23\% of newborns. The low values of amplitudes, typical for this period, justify the use of Eq. (5).

A similar picture was observed in the United Kingdom during the same period. In England and Wales in 1960-1976 (vaccination there reached a high level in 1957) there were inter-epidemic periods of about 3-4 years (an average of 180 weeks or 41.5 parasite generations cycles) (Anderson and May, 1991; Anderson et al., 1984), and the actual birth rate was about 20 births per thousand population (US Bureau of Census, 2002), or about $b=0.00167$. Hence, the effective vaccination rates in the United Kingdom in this period were also about $23 \%$ of newborns.

After 1980, the inter-epidemic periods of pertussis in New Zealand increased further. Epidemics occurred in 1974, 1978, 1982, 1986, 1991, 1996 and 2000-2001 (Cullen and Walker, 1997; NZ ESR, 1997), that is for this period the inter-epidemic period extended from 4 (48 parasite generations) to 5 (60 parasite generations) years. After 1976, the birth rate dropped to 17 births per thousand population per year on average (NZ Yearbook, 1998, p. 92), or $b=0.00142$. However, this lengthening of the epidemic cycle cannot be attributed to the birth rate decline alone. For the 5 years cycles, $T_{n}=60$, the apparent rate of new susceptibles influx $\hat{b}=0.00069$, or the rate of effective vaccination of about $52 \%$. If we take $T_{n}=56$ as an average value between 48 (4-year cycle) and 60 (5-year cycle) parasite generations, from Eq. (5) we obtain the apparent rate of new susceptibles influx $\hat{b}=0.00079$. This value gives the effective vaccination rate of $44 \%$.

In reality, however, this percentage is lower: the interepidemic period grows with amplitude, and after 1980 amplitudes of oscillations have been gradually increasing. Judging by the number of hospitalizations, the ratio $I_{\max } / I_{\min } \approx 13$ for the 1996 epidemic (Cullen and Walker, 1997; NZ Health Information Service, 2000). In fact this value is even bigger since, instead of $I_{\max }$ and $I_{\min }$ values, the total numbers of hospitalizations for 1994 and 1996 have been taken, and also pertussis is known to be grossly underdiagnosed (Deeks et al., 1999). For the period since 1980 , the ratio $I_{\max } / I_{\min }>20$ can be considered as realistic in New Zealand. Table 2 gives values of the inter-epidemic periods for $\rho=0.015$ (which corresponds with the apparent birth rate $\hat{b}=$ 0.00094 , or with the actual birth rate $b=0.00142$ at the rate of effective vaccination of $33 \%$ ). At $I_{\max } / I_{\min } \approx 20$, Table 2 gives $T_{n} \approx 56$, or a $4 \frac{1}{2}$-year cycle. Further, for $\rho=0.013$, or for the actual birth rate $b=0.00142$ at the rate of effective vaccination $\hat{p} \approx 0.43$, or $43 \%$, the computations give that $T_{n} \approx 60$ at $I_{\max } / I_{\min } \approx 20$.
Table 2

Period $T_{n}$ vs. amplitudes for $\rho=0.015$

\begin{tabular}{lcllll}
\hline$I_{\min }$ & $\frac{I_{\max }}{I^{*}}$ & $T_{n}$ & $\frac{I_{\min }}{I^{*}}$ & $\frac{I_{\max }}{I_{\min }}$ & $T_{n}$ \\
\hline 0.002 & 4185 & 73 & 0.22 & 12.5 & $54-55$ \\
0.004 & 1890 & $70-71$ & 0.24 & 11 & 54 \\
0.006 & 1180 & 69 & 0.26 & 9.8 & 54 \\
0.008 & 840 & 68 & 0.28 & 8.75 & 54 \\
0.01 & 651 & 67 & 0.30 & 7.9 & $53-54$ \\
0.02 & 283 & 64 & 0.35 & 6.2 & 53 \\
0.03 & 173 & 62 & 0.40 & 5.0 & $52-53$ \\
0.04 & 121 & 61 & 0.45 & 4.2 & $52-53$ \\
0.05 & 91.6 & 60 & 0.50 & 3.5 & 52 \\
0.06 & 72 & $59-60$ & 0.55 & 3 & 52 \\
0.07 & 59.3 & 59 & 0.60 & 2.58 & 52 \\
0.08 & 50 & 58 & 0.65 & 2.25 & 52 \\
0.09 & 42.7 & 58 & 0.70 & 1.97 & $51-52$ \\
0.10 & 37.1 & 57 & 0.75 & 1.73 & $51-52$ \\
0.12 & 29.2 & 57 & 0.80 & 1.54 & $51-52$ \\
0.14 & 24.6 & 56 & 0.85 & 1.38 & $51-52$ \\
0.16 & 19.6 & $55-56$ & 0.90 & 1.24 & $51-52$ \\
0.18 & 16.7 & 55 & 0.95 & 1.1 & $51-52$ \\
0.20 & 14.3 & 55 & 0.999 & 1.002 & \\
\hline
\end{tabular}

\section{Discussion and conclusion}

The model applied here appears extremely simple, even oversimplified. Nevertheless, it provides reliable estimation of the inter-epidemic period. Furthermore, the inter-epidemic period appears to be a robust invariant, and there is no specific limitation on the type of model applied.

We neglected seasonal effects: seasonality would very likely be annual (as in measles) adjusting the epidemic cycle to the seasons and forming an epidemic cycle of integer number of years. That is the seasonal effects are on a much shorter time-scale than the observed periodicities which would therefore very likely be unaffected.

The obtained figures indicate that in New Zealand the effective vaccination rate against pertussis is lower than $50 \%$, and perhaps even as low as $33 \%$ of the population. These figures contradict the medical statistics which claim that more than $80 \%$ of the newborns in New Zealand are vaccinated against pertussis (Turner et al., 2000). This contradiction is due to the mentioned unreliability of the available vaccine. The fact that the fraction of immune population obtained here is considerably lower than the fraction of vaccinated population implies a high level of vaccination failure.

We believe that we can safely conclude that under the current conditions (with the present vaccine and the current vaccination practice) the effective vaccination rate against pertussis in New Zealand is considerably lower than is expected. Based on the present data it is perhaps lower than $50 \%$ of the population. 


\section{References}

Anderson, R.M., May, R.M., 1991. Infectious Diseases in Humans: Dynamics and Control. Oxford University Press, Oxford.

Anderson, R.M., et al., 1984. Oscillatory fluctuations in the incidence of infectious disease and the impact of vaccination: time series analysis. J. Hyg. Cambridge 93, 587-608.

Bartlett, M.S., 1957. Measles periodicity and community size. J. R. Stat. Soc. A 120 (1), 48-70.

Bartlett, M.S., 1960. The critical community size for measles in the United States. J. R. Stat. Soc. A 123 (1), 37-44.

Cullen, R.M., Walker, W.J., 1996. Measles epidemics 1949-91: the impact of mass immunisation in New Zealand. N. Z. Med. J. 109, 400-402.

Cullen, R.M., Walker, W.J., 1997. Pertussis hospitalizations and mass vaccination in New Zealand 1948-1996. N. Z. Fam. Physician 24 (6), 45-48.

Cullen, R.M., et al., 1999. A model of measles endemicity. Nonlinear Anal. 35, 191-198.

Cullen, R.M., et al., 2003. Seasonality and critical community size for infectious diseases. ANZIAM J. 44, 501-512.

Deeks, S., et al., 1999. Failure of physicians to consider the diagnosis of pertussis in children. Clin. Infect. Dis. 28, 840-846.
Dietz, K., 1976. The incidence of infectious diseases under the influence of seasonal fluctuations. Lect. Notes Biomath. 11, $1-15$.

Keeling, M.J., Grenfell, B.T., 1997. Disease extinction and community size: modeling the persistence of measles. Science 275, 65-67.

Murray, J.D., 1993. Mathematical Biology. Springer, Berlin.

New Zealand Official Yearbook, 1998. GP Publications, Wellington, 1998.

NZ ESR, 1997. Annual Surveillance Summary.

NZ Health Information Service, 2000. Statistics at glance: pertussis, number of hospitalisations, 1989-1999. URL: http://www.nzhis.govt.nz/stats/diseases/pertussis.html

NZ Ministry of Health, 2001. Surveillance and control notes. N. Z. Public Health Rep. 8(5), 36-37.

Soper, H.E., 1929. Interpretation of periodicity in disease-prevalence. J. R. Stat. Soc. 92, 34-73.

Tu, P.N.V., 1994. Dynamical Systems. Springer, Berlin.

Turner, N., et al., 2000. Improving immunisation coverage: what needs to be done? N. Z. Public Health Rep. 7 (3/4), 1-4.

US Bureau of the Census, 2002. International Data Base (on line).

US Department of Human Services, 1994. Summary of Notifiable Diseases, United States, 1993. 